\title{
PROCESSING OPTICAL AND SAR DATA FOR BURNED FORESTS MAPPING: AN INTEGRATED FRAMEWORK
}

\author{
Daniela Stroppiana $^{(1)}$, Ramin Azar ${ }^{(1)(2)}$, Fabiana Calò ${ }^{(3)}$, Antonio Pepe ${ }^{(3)}$, Pasquale Imperatore ${ }^{(3)}$, \\ Mirco Boschetti $^{(1)}$, João M. N. Silva ${ }^{(4)}$, Pietro A. Brivio ${ }^{(1)}$, and Riccardo Lanari ${ }^{(3)}$ \\ ${ }^{(1)}$ IREA-CNR, Via Bassini 15, 20133 Milano, Italy; \\ Email: stroppiana.d@irea.cnr.it; azar.r@irea.cnr.it, boschetti.m@irea.cnr.it, brivio.pa@irea.cnr.it
}

\author{
${ }^{(2)}$ Politecnico di Milano, Dipartment of Civil and Environment Engineering, Piazza L. da Vinci, 32, Milano, Italy. \\ (3) IREA-CNR, Via Diocleziano 328, 80124 Napoli, Italy; \\ E-Mail:calo.f@irea.cnr.it,pepe.a@irea.cnr.it,imperatore.p@irea.cnr.it, lanari.r@irea.cnr.it
}
${ }^{(4)}$ University of Lisbon, School of Agriculture, Forest Research Centre, Tapada da Ajuda, 1349-017 Lisboa, Portugal; E-mail: joaosilva@isa.ulisboa.pt

\begin{abstract}
The application of an integrated monitoring tool to assess and understand the effects of annually occurring forest fires is presented, with special emphasis to Mediterranean and Temperate Continental zones of Europe. The distinctive features of the information conveyed by optical and microwave remote sensing data have been firstly investigated, and pertinent information have been subsequently combined to identify burned areas at the regional scale. We therefore propose a fuzzy-based multisource framework for burned area mapping, in order to overcome the limitations inherent to the use of only optical data (which can be severely affected by cloud cover or include low albedo surface targets). The relevant experimental validation has been carried out on an extensive area, thus quantitatively demonstrating how our approach successes in identifying areas affected by fires. Furthermore, the proposed methodological framework can also be profitably applied to ESA Sentinel (optical and SAR) data.
\end{abstract}

\section{INTRODUCTION}

Forest fires in the Mediterranean and Temperate Continental zones of Europe have strong ecological (soil erosion, hydrological cycle, etc.) and socioeconomic (economic damage, loss of human lives, etc.) impacts. Hence, to assess and understand the impact of annually-occurring forest fires, improved and integrated monitoring tools are of crucial importance. For studying forest fires, and more specifically burned areas, the different methodologies and techniques that have been developed can be categorized according to the investigated spectral portion (visible, infrared and microwave) of the electromagnetic spectrum.

In order to achieve the goal of generating burned area map products at a regional level, the most commonly adopted practice is the use of optical images. However, the effects of the spectral confusion between burned areas and low-albedo surfaces typically hamper achieving reliable results with optical data, only. The aim of this paper is to investigate how the distinctive features of the information conveyed by optical and SAR data can be combined to identify burned areas. Specifically, an integrated framework for burned area maps production based on a fuzzy algorithm has been developed. This framework is an extension of the automated algorithm originally proposed for Landsat TM/Enhanced TM plus (ETM+) data [1]. The approach relies on the use of fuzzy sets theory and a region growing algorithm, for the interpretation and integration of spectral indices and scattering signature. As a result, the scattering signature is essentially treated as an additional information to be properly ingested into the fuzzy algorithm. Thus, this approach allows us to exploit the complementary features of optical and SAR information for burned area mapping into an integrated multi-source framework.

The main emphasis in this paper is on Mediterraneantype regions; in particular, our analysis is focused on Portugal, which has been affected by remarkable fire events during 2003. For such a purpose, a multitemporal analysis (pre-fire and post-fire acquisitions) has been carried out by employing both Landsat5 TM and (C-band) ENVISAT ASAR data. We have exploited, as a reference, a fire atlas available over Portugal, covering the period from 1975 to 2009 and derived from Landsat imagery. It is worth remarking that Portugal has been considered as a test site because it is one of the few countries having operational programs to systematically map burned areas. The burned area maps obtained by applying the fuzzy algorithm have been compared with the reference data extracted from the fire atlas, in order to evaluate the performance of our approach. 


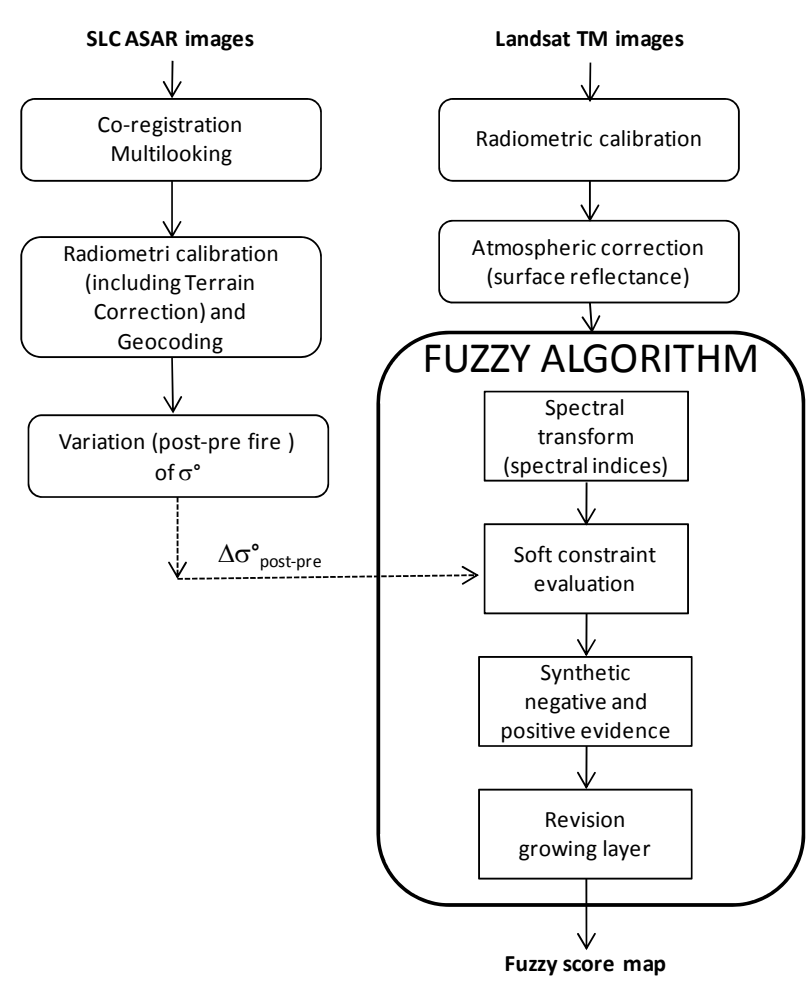

Figure 1. Flowchart of the pre-processing and classification steps implemented for the integration of SAR and optical data in the fuzzy burned area mapping algorithm.

\section{METHODOLOGY}

The adopted approach relies on the use of fuzzy sets theory for the interpretation and integration of spectral indices and scattering signature [1],[3] and a region growing algorithm. This approach is theoretically based on the concept of the reinforcement of burn evidence [1]: spectral indices derived from the multi-spectral images acquired by optical satellite sensors can provide consistent and coherent information over burned areas, whereas they may have a complementary, incoherent behavior over confusing surfaces (e.g. cloud and topographic shadows, low density unburned vegetation, wetlands). Specifically, six spectral indices are taken into account (CSI, EVI, EVI2, MIRBI, NBR, NBR2, NDVI, SAVI) [3]. The evidence of burn given by each index (i.e., partial positive evidence) is quantified by using fuzzy membership functions. Similarly, two optical indices (NBR and MIRBI) have been exploited to characterize the unburned surface, and fuzzy membership functions have been defined to compute the negative evidence (i.e., evidence of unburned conditions). The map of backscattering variation across a fire-event $\left(\sigma_{\text {post-pre }}^{\circ}\right)$ is computed starting from the preand post-fire sigma naught $\left(\sigma^{\circ}\right)$ maps [2], and a fuzzy membership function on the domain of the backscattering variation is defined to extract partial negative evidence to be subtracted to the synthetic positive evidence obtained from the optical spectral indices (see Fig.1). Therefore, the fuzzy burned area mapping algorithm relies on the definition of the membership functions of fuzzy sets for the input optical and SAR features (i.e. the optical spectral indices and $\sigma^{\circ}$ ) to determine the degrees of partial positive/negative evidence (fuzzy scores). Partial positive and negative evidence brought by the optical and SAR data have been integrated by using Ordered Weighted Averaging (OWA) operators $[4,5]$ and a region growing algorithm has been subsequently applied. For a more detailed description the reader is referred to [3].

\section{MULTITEMPORAL ANALYSIS ON AN EXTENSIVE SCENARIO}

The results obtained by applying the proposed approach, in order to demonstrate its validity, to a concrete operative scenario are now provided.

\subsection{Study area and dataset description}

We have conducted an analysis including almost entire Portugal, in order to validate the proposed approach. Portugal is one of the European countries most affected by fires; nonetheless, the number of fires and the burned areas exhibit a large temporal and spatial variability. In particular, this country was affected by severe forest fires during summer 2003. Moreover, it is worth mentioning that Portugal has been considered because it is one of the few countries having operational programs to systematically map burned areas. For the purposes of our multi-temporal analysis, we have employed Landsat5 TM and (C-band) ENVISAT ASAR data, both acquired during the year 2003. Five Landsat-5 TM scenes were acquired on clear sky dates in late summer 2003 (see Figure 2.a). SAR (VV polarization) images have been collected over the available nine ENVISAT ASAR data frames shown in Fig. 2.b, including both pre-fire and post-fire acquisitions. We have also exploited, as a reference, a fire atlas available over Portugal, derived from Landsat imagery.

\subsection{Experimental results}

SLC SAR images have been co-registered and then averaged by performing a multi-look operation with three looks in range direction and fifteen looks in the azimuth one, resulting in a multilooked SAR pixel spacing of about $60 \mathrm{~m} \times 80 \mathrm{~m}$. Afterwards, multi-looked amplitude SAR images have been properly geocoded, and radiometrically calibrated [5] to obtain sigma naught $\left(\sigma^{\circ}\right)$ maps, with a $60 \mathrm{~m} \times 80 \mathrm{~m}$ spatial resolution on the ground. 


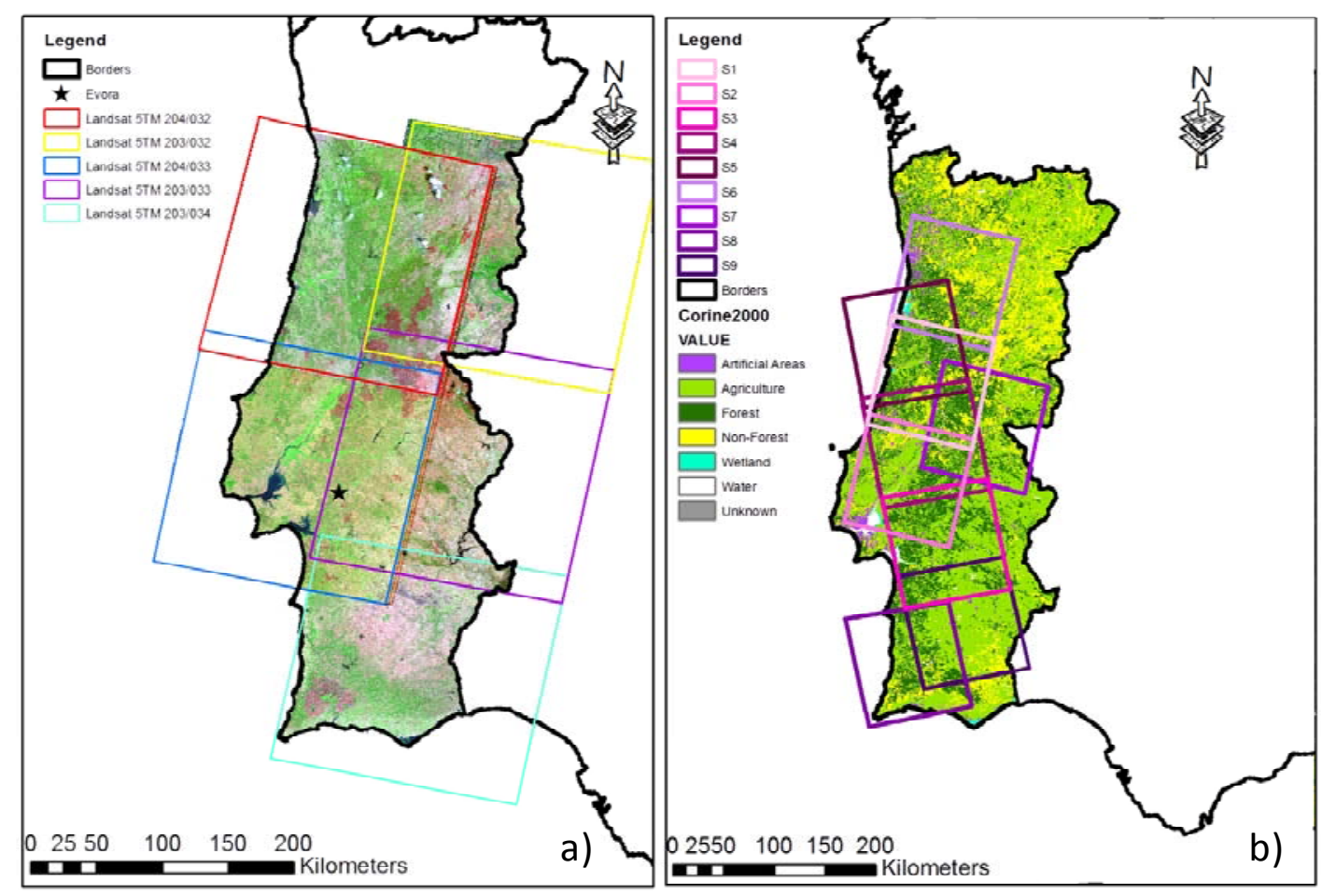

Figure 2. Study area showing footprints of the considered optical and SAR images, acquired during the year 2003: a) Landsat-5 TM images shown as false color composite mosaic (R-G-B: 5-4-3); b) ENVISAT ASAR data frames overlaid on the Corine Land Cover (CLC2006) map.

To accurately perform the calibration process of SAR images amplitude, a terrain height correction step has been implemented by exploiting the satellite orbital information and ASTER GDEM (Advanced Spaceborne Thermal Emission and Reflection Radiometer Global Digital Elevation Model) with $30 \mathrm{~m}$ spatial resolution. Notice that the ASTER GDEM is the only DEM at 1 arc-second (approximately $30 \mathrm{~m}$ ) grid with global coverage. The NASA SRTM DEM 1 arc-second data coverage is freely available only for the United States. On the contrary, 3 arc-second data are available for worldwide coverage. In particular, ASTER GDEM has been checked for artefacts and the minimum and maximum values occurring in the study areas have been evaluated. The temporal variation of backscattering $\left(\Delta \sigma_{\text {post-pre }}^{\circ}\right)$ for the 2003 season has been computed starting from the pre- and post-fire (geocoded) $\sigma^{\circ}$ maps over the available nine SAR data frames.

To assess the ability of the proposed integrated framework to map burned areas accurately, the fuzzy score map has been analyzed (Fig. 3.a): color keys represent potentially burned areas, taking values in the range $[0,1]$. The reference burned areas extracted from the Fire Atlas of Portugal are also shown in Fig. 3.b and have been used to validate our approach.

It is worth remarking that our results (Fig3.a) turn out in good agreement with the reference map (Fig3.b). Noticeably, it can be observed (see Fig3.a) that burned areas are identified by relatively high (yellow to red colors) fuzzy scores, while low scores correspond to areas that are unlikely to be burned. A binary burned area map (0-unburned, 1-burned) can be derived by applying a threshold to the fuzzy score map [3]. By assessing the accuracy of the burned/unburned area maps as a function of the threshold applied to the fuzzy score map, we found that errors vary between two extremes: a map where areas identified as burned are highly reliable, i.e. highest user's accuracy (commission error $<5 \%$ ) and a map where almost all actually burned areas are identified, i.e. highest producer's accuracy (omission error $<10 \%$ ). An intermediate case, with a 0.5 threshold value, leads to commission and omission errors of about $20 \%$ over the study area.

In addition, in order to show the importance of integrating optical and microwave data, we have also analyzed some cases in which poor results are obtained by using only optical information (due to the resulting spectral confusion between burned areas and low albedo surfaces). In such circumstances, whenever optical and microwave data are jointly employed, a significant reduction of the commission error is obtained. The analysis of the contribution of SAR data to the accuracy of the final burned area map was carried out at the regional and local scales. Results show that $\Delta \sigma^{\circ}$ images introduced into the fuzzy algorithm can correct for erroneously identified burned areas and thus reduce commission errors. This effect is particularly 

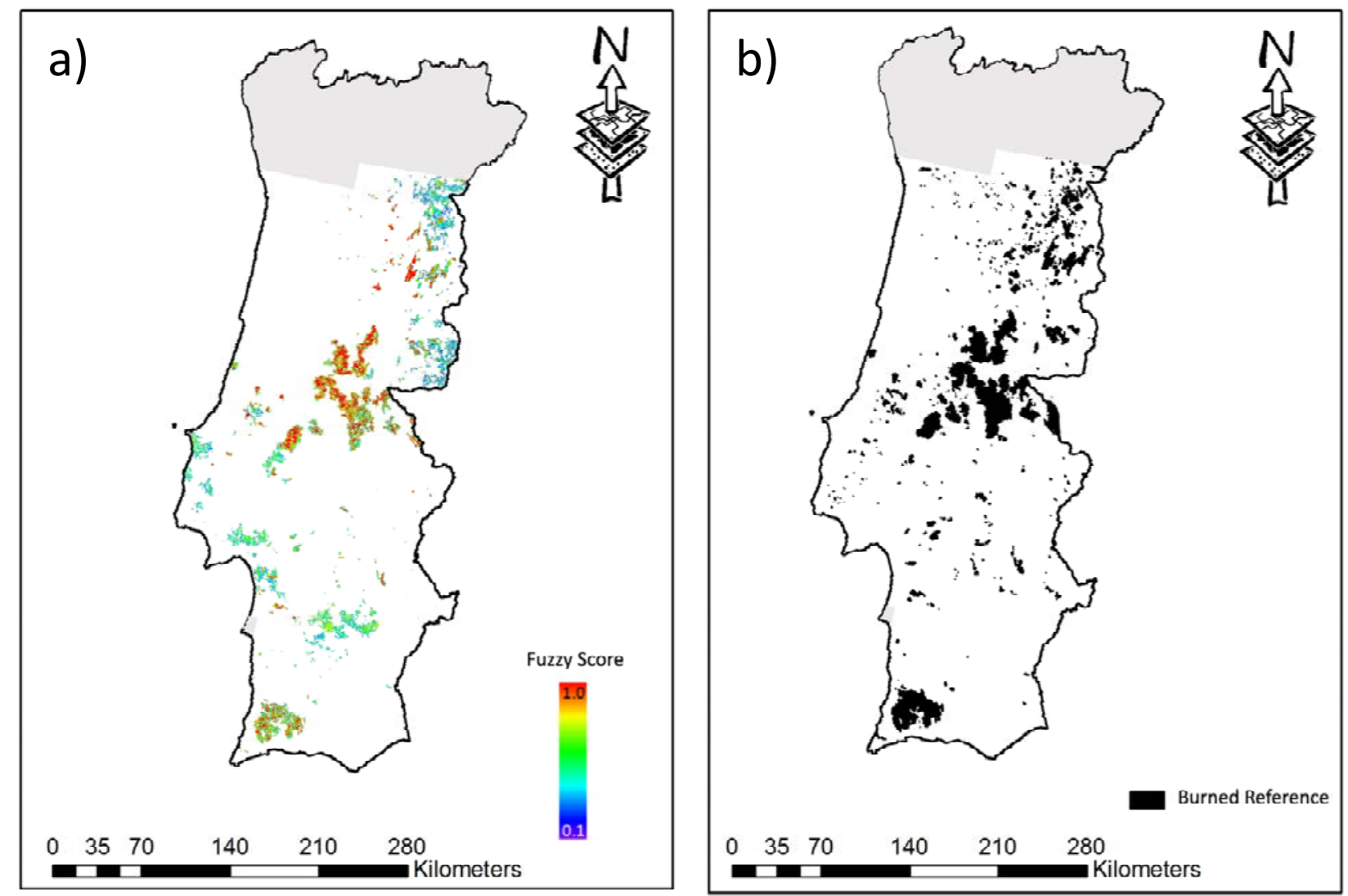

Figure 3. a) The fuzzy score map output from the fuzzy algorithm; b) the reference map derived from the Fire Atlas of Portugal (black and white represent burned and unburned areas, respectively).

evident when clear sky optical images are not available (high incidence of could shadows) and where low albedo surfaces (e.g. wetlands) are present and not masked out with external information on the land cover. As a consequence, the contribution of the optical + SAR integration can be significant.

\section{CONCLUSIONS}

As a result, the proposed methodological framework, by integrating optical and SAR data, successes in identifying forested areas affected by fires. In addition, the complementary character of the information conveyed by optical and SAR data also permits to achieve reduced error in remarkable critical cases: e.g., the optical data are severely affected by cloud cover or include low albedo surface targets that are likely to be confused with burned areas (e.g. cloud shadows). The proposed methodology also offers a suitable framework for ESA Sentinel (optical and SAR) data exploitation.

\section{REFERENCES}

1. D. Stroppiana, G. Bordogna, P. Carrara, M. Boschetti, L. Boschetti, P.A. Brivio, "A method for extracting burned areas from Landsat
TM/ETM+ images by soft aggregation of multiple Spectral Indices and a region growing algorithm," ISPRS J. Photogramm. 2012, 69, pp. 88-102.

2. F. T. Ulaby, R. K. Moore, A. K. Fung, Microwave Remote Sensing. Reading, MA: Addison-Wesley, 1982.

3. D. Stroppiana, R. Azar, F. Calò, A. Pepe, P. Imperatore, M. Boschetti, J. M. N. Silva, P.A. Brivio, and R. Lanari, "Integration of optical and SAR data for burned area mapping in Mediterranean regions”, Remote Sens. vol. 7, no. 2, pp.1320-1345, January 2015.

4. R. R. Yager, "On ordered weighted averaging aggregation operators in multicriteria decision making”. IEEE Trans. Syst. Man Cyb. 1988, 18 (1), 183-190.

5. Small, D.; Schubert, A.; Rosich, B.; Meier, E. Geometric and Radiometric Correction of ESA SAR Products. In Proceedings of the ENVISAT Symposium 2007, Montreux, Switzerland, 23-27 April, 2007. 